\title{
Design of A Novel Foil for Millimeter Wave Application
}

\author{
Jin Cheng ${ }^{1, a^{*}}$, Hu Yang ${ }^{1, b}$, Shaojun Mao, ${ }^{2, c}$, Lei $\mathrm{Gu}^{2, \mathrm{~d}}$ and Fei Zhao ${ }^{2, \mathrm{e}}$ \\ ${ }^{1}$ College of Electronic Science and Engineering, National University of Defense Technology \\ Changsha410073, Hunan province, China \\ ${ }^{2}$ Southwest Electronics and Telecommunication Technology Research Institute \\ Chengdu610041, Sichuan province, China \\ achengjin9380@163.com
}

Keywords: millimeter wave, foil, radar cross section

Abstract. In order to lengthen floating time of foil in the air and improve its scattering abilities, this paper proposes a novel structure of millimeter wave foil. By cutting useless part of foil, its weight is reduced appropriately while its scattering properties as well as floating time are strengthened which usually means effective interference.

\section{Introduction}

Chaff is used as a material in radar passive interference and half wave-length chaff is the commonest one. However, millimeter-wave chaff is rather small in size which increases difficulties in cutting, packaging and controlling of its spatial orientations. What's worse, radar cross section(RCS) of single chaff is relatively low; therefore, numerous chaffs are usually needed to compose chaff clouds in applications. In this case, large quantities make it difficult to disperse quickly which exacerbates shielding effect and limits interfering effect of chaff clouds.

Foil is the most efficient passive interference against millimeter-wave radar and reacts quickly in actual application [1]. In the process of manufacture, millimeter wave foil is much bigger in size than chaff, avoiding the difficulties in cutting and packaging preferably. In electromagnetic properties, foil has an advantage in operating bandwidth, which means continuous interfering in frequency. However, larger size means higher weight and shorter floating time in the air, which reduces the working time of effective interfering. Therefore, researches on foil are of practical significance in passive jamming technology.

Researches on the foil have been investigated deeply recently. Paper [2] uses approximate method to obtain the formula of circular foil's RCS and explores its application. Paper [3] uses the physical optics to calculate scattering characteristics of single foil and studies foil's frequency characteristics of average RCS. Paper [4] has conducted the research on the mixture interference of chaff and foil in $3 \mathrm{~mm}$ wave band and proved the effective of mixture interference. Paper [5] changes each parameter of foil and points that different factors affect foil's scattering ability in varying degrees. However, these studies mainly focused on the analysis of foil's RCS, rarely refer to foil's structure design.

This paper begins from the scattering analysis of conventional foil. Comparison between results of approximate solution and simulation is conducted, finding that the two results correspond to each other well, which indicates the effective of simulation to some extent. Finally, a new structure on millimeter wave foil is proposed by cutting useless part of the foil, which reduces weight and enhances scattering properties.

\section{Scattering properties of conventional foil}

RCS is the most important index for a foil, which represents target's scattering properties. General formula of RCS is given by Eq.1 [6]: 
$\sigma=4 \pi R^{2}\left(\frac{E^{\prime}}{E}\right)^{2}$

Where $R$ is the distance between the source and scatterer, $E$ is the electric field intensity of incident wave, $E^{\prime}$ is the electric field intensity of scattering wave.

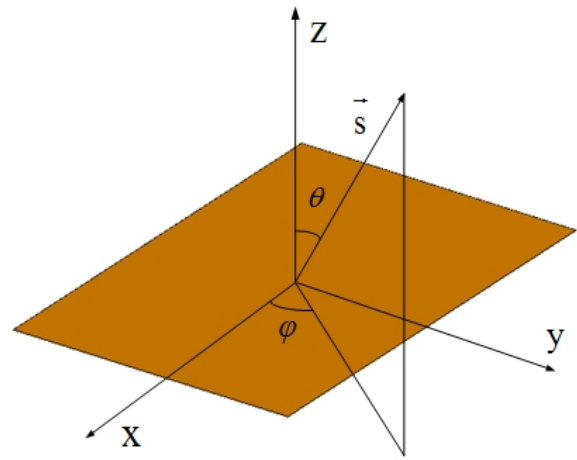

Fig. 1 The foil's scattering characteristics

According to the scattering situation, a coordinate system is set, shown in Fig.1. The rectangular foil has lengthes of $a$ and $b$ in width, $(\theta, \varphi)$ is incident angle. Rectangular foil's RCS can be given by Eq.2 [2]:

$$
\sigma=\frac{4 \pi a^{2} b^{2}}{\lambda^{2}} \cos ^{2} \theta\left[\frac{\sin \left(\frac{2 \pi a \sin \theta}{\lambda}\right)}{\frac{2 \pi a}{\lambda} \sin \theta}\right]^{2}
$$

Where $\lambda$ is the wavelength, $\theta$ is the angle between incident wave and foil's vertical direction, polarization direction is parallel to the edge of $a$.

Set operating frequency being $35 \mathrm{GHz}$ and assume foil as a square with $17.2 \mathrm{~mm}$ in width with its thickness going to zero. Comparison between results of approximate solution and simulation are shown in Fig.2 and Fig.3.

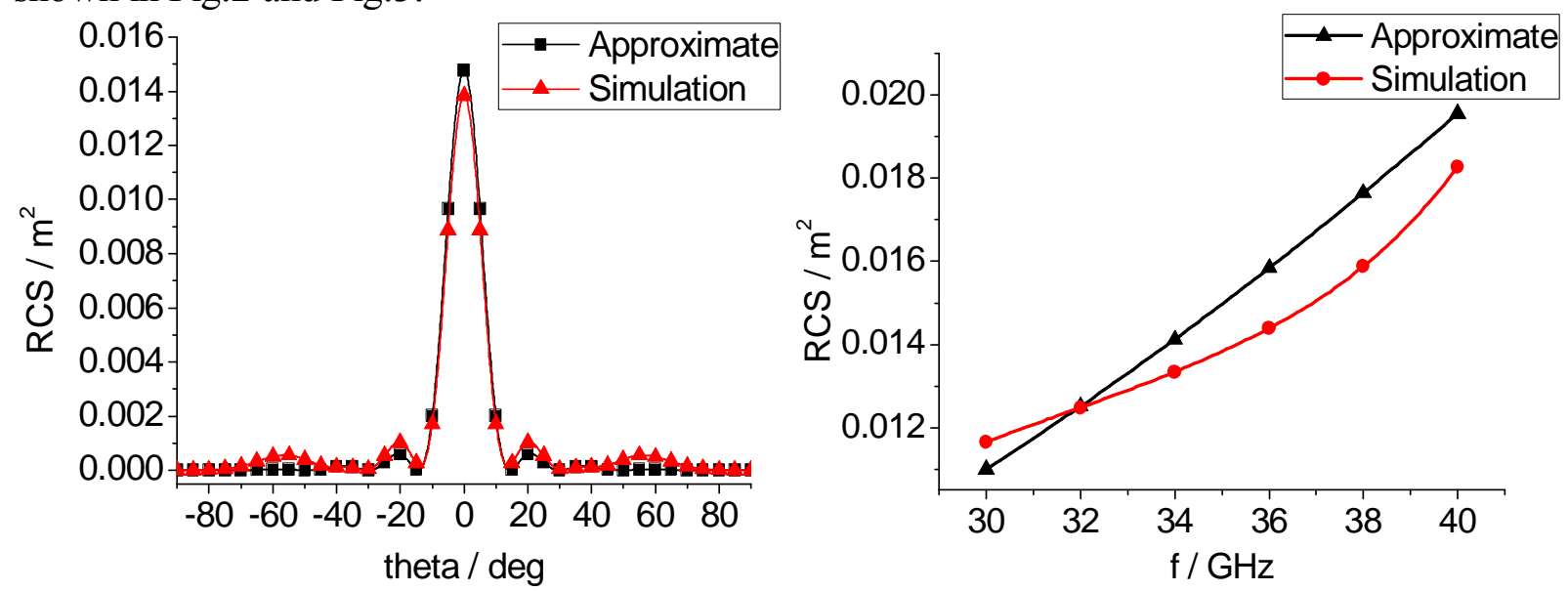

Fig.2. RCS in different incident angles

Fig.3. RCS in different frequencies

Fixing working frequency and changing the incident angle $\theta$, the RCS to incident angle is shown in Fig.2. Fixing incident angle $\theta=0^{0}$ and changing the working frequency, the RCS to frequency is shown in Fig. 3.

As it can be seen from Fig. 2, the RCS of simulation and approximate solution both reach maximum at the direction of $\theta=0^{0}$ and decrease sharply with the incident angle changed. When $\theta=90^{\circ}$, $\mathrm{RCS}$ reaches its minimum. In total, the results of two types are same in tendency. However, difference of RCS in amplitude occurres clearly at $\theta=0^{\circ}$, which is caused by the neglection of edge diffraction in 
the process of educating approximate formula while simulation result take it into consideration based on the actual situation.

In Fig. 3, both approximate solution and simulation indicate that foil is a kind of wideband interference, which RCS increases with frequency steadily. At the same time, the errors between approximate solution and simulation are acceptable which means simulation values are credible. Thus, numerical simulation method can be used in designing novel foil and testing foil's characteristics.

\section{Novel structure of millimeter wave foil}

Because of foil's large size, its weight is much higher than chaff, which decreases foil's floating time in the air and weakens the interfering effects as a result. Therefore, our purpose is to reduce foil's weight while maintain its scattering properties.

In order to reduce the weight of foil, a simple solution is cutting out some useless part, which contributes few to scattering properties according to the distribution of induced current on the surface of foil. At the same time, this change does not affect the scattering properties of the whole. Thus, we can reduce foil's weight and achieve our aim of design.

Take a square with length of $\lambda / 2$ as a basic unit. Cut out some part on each basic unit according to the principle mentioned above and the structure will be obtained, as is shown in Fig. 4.

In Fig.4, the interception area is a square with Length $L=2 \mathrm{~mm}$, locating at the center of each basic unit. For the novel strtucture, 16 interception areas are cutted out with the weight of reduction accounting to $21.6 \%$ in total. Therefore, weight of foil reduces large relatively compared with conventional foil.

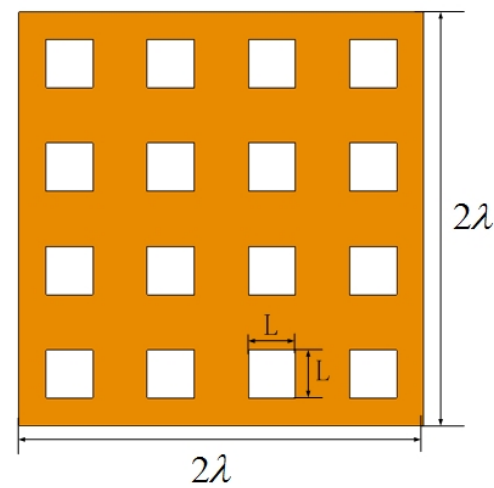

Fig. 4 A novel structure on millimeter wave foil

Keeping frequency constant, RCS of the novel foil changes with incident angles, as is shown in Fig.5. Keeping incident angle $\theta=0^{0}$, RCS of the novel foil changes with frequencies, as is shown in Fig.6.

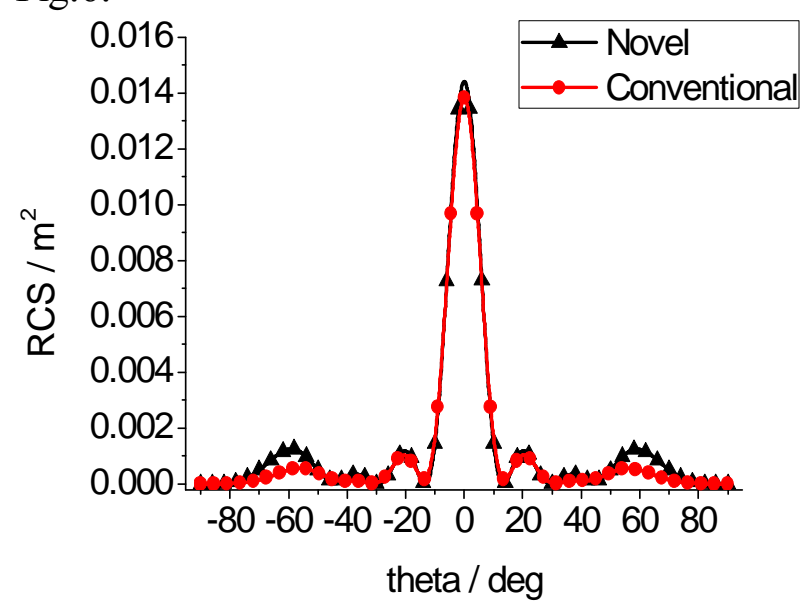

Fig. 5 RCS in different incident angles

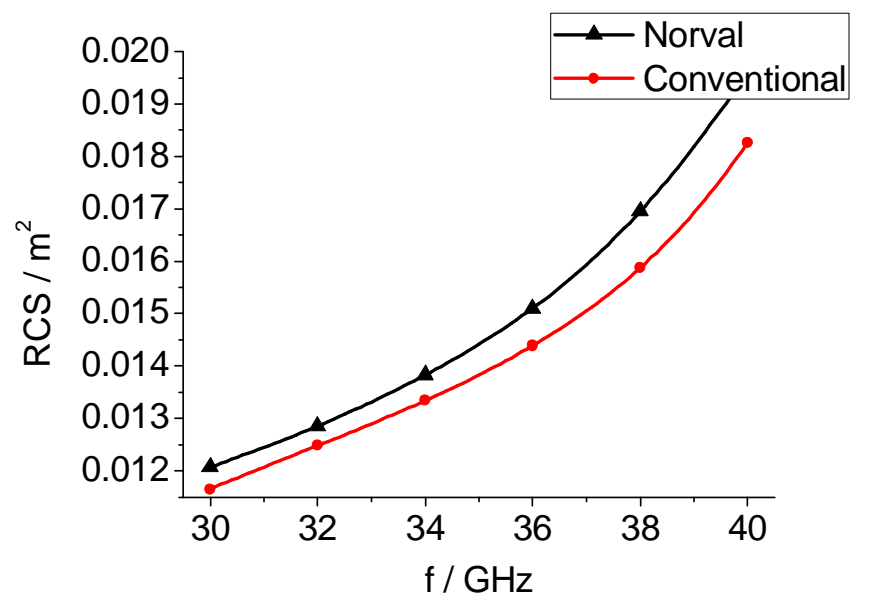

Fig. 6 RCS in different frequencies 
As is shown in Fig. 5, RCS of the novel foil is a little higher than that of conventional foil in the region $\theta=60^{\circ}$ and especially in $\theta=0^{\circ}$. Therefore, the scattering properties of the novel foil have been improved to some extent. In Fig. 6, the maximum RCS of the novel foil keeps rise within $30 \mathrm{GHz} \sim 40 \mathrm{GHz}$ which is higher than that of conventional foil as well. Thus, the novel structure maintains the property of wideband interfering.

Accordingly, the novel foil reduces its weight, enhances its scattering ability and maintains wideband interfering characteristics at the same time which reaches our design goals and is functional effective.

\section{Conclusion}

Considering the disadvantages of big weight and short floating time of foil, a novel form of foil structure is designed in engineering by cutting out some useless part of basic unit. This foil reduces its weight appropriately so as to lengthen its floating time in the air. What's more, this structure improves foil's scattering abilities and gets a better jamming effects. It should be pointed out that this design can be expanded based on actual apply.

\section{References}

[1] Zhenxing Jin. The Application of Chaff and Foil in the Millimeter Wave Radar EW, J. Electronic Warfare, 2012,4(145): 33-36.

[2] Jin Chen. The Circular Metal Chaff and its Application, J. Electronic Warfare, 2012,4(145):33-36. In Chinese.

[3] Jinliang Li. Frequency characteristics of foil average RCS, J. Aerospace Electronic Warfare, 2009,25(4) : 39- 41. In Chinese.

[4] Huaichao Cui. Jamming Analysis of Chaff and Foil on Three Millimeter Waves, J. Ordnance Industry Automation, 2011,30(8) : 15-17.

[5] Haiqi Wang. Research on Preparation Technology of Combustible foil and IR/MMW Character. Nanjing : Nanjing University of Science and Technology, January, 2013. In Chinese.

[6] Jin Chen. Principles of Radar Chaff Jamming. Beijing: National Defense Industry Press, 2007,05. In Chinese. 